Cahiers $d u$ MONDE RUSSE

\section{Cahiers du monde russe}

Russie - Empire russe - Union soviétique et États indépendants

48/2-3 | 2007

Les résonances de 1905

\title{
Les historiens russes et la révolution de 1905
}

\section{KORINE AMACHER}

\section{OpenEdition \\ Journals}

Édition électronique

URL : https://journals.openedition.org/monderusse/9023

DOI : $10.4000 /$ monderusse. 9023

ISSN : $1777-5388$

Éditeur

Éditions de l'EHESS

Édition imprimée

Date de publication : 15 avril 2007

Pagination : 499-518

ISBN : 978-2-7132-2147-7

ISSN : $1252-6576$

Référence électronique

KORINE AMACHER, "Les historiens russes et la révolution de 1905 », Cahiers du monde russe [En ligne], 48/2-3 | 2007, mis en ligne le 01 janvier 2007, consulté le 02 septembre 2022. URL : http:// journals.openedition.org/monderusse/9023; DOI : https://doi.org/10.4000/monderusse. 9023 
chercher : repérer : avancer

Cet article est disponible en ligne à l'adresse :

http://www.cairn.info/article.php?ID REVUE=CMR\&ID NUMPUBLIE=CMR 482\&ID ARTICLE=CMR 4820499

Les historiens russes et la révolution de 1905

par KORINE AMACHER

Editions de I'EHESS | Cahiers du monde russe

2007/2-3 - Vol 48

ISSN 1252-6576 | ISBN 9782713221477 | pages 499 à 518

Pour citer cet article :

-AMACHER K., Les historiens russes et la révolution de 1905, Cahiers du monde russe 2007/ 2-3, Vol 48, p. 499-518.

Distribution électronique Cairn pour les Editions de l'EHESS.

(C) Editions de l'EHESS. Tous droits réservés pour tous pays.

La reproduction ou représentation de cet article, notamment par photocopie, n'est autorisée que dans les limites des conditions générales d'utilisation du site ou, le cas échéant, des conditions générales de la licence souscrite par votre établissement. Toute autre reproduction ou représentation, en tout ou partie, sous quelque forme et de quelque manière que ce soit, est interdite sauf accord préalable et écrit de l'éditeur, en dehors des cas prévus par la législation en vigueur en France. Il est précisé que son stockage dans une base de données est également interdit. 


\section{LES HISTORIENS RUSSES ET LA RÉVOLUTION DE 1905}

La révolution de 1905 a eu une influence profonde sur la vie politique et socioculturelle de la Russie. Le domaine des recherches historiques est à cet égard révélateur. En effet, l'évolution des formes d'action politique, la création des partis ont renforcé les possibilités d'organisation sociale et politique. Fortement sollicités par l'actualité de leur époque, les historiens ne sont pas restés hors de ce processus. Les événements qui secouèrent la Russie durant ces années ont suscité une radicalisation politique, parfois un véritable engagement politique d'historiens auparavant plus modérés ou peu politisés. Dès 1905, nous observons une cristallisation des «passions » politiques, conduisant parfois à de nouvelles interprétations historiques. La révolution de 1905 a ainsi amené certains historiens à donner sens au passé à la lumière des événements présents, voire à réorienter leurs recherches, en raison de l'émergence de nouvelles thématiques historiques. Celles-ci ont pu être étudiées par les chercheurs qui avaient désormais accès à des documents et à des archives auparavant inaccessibles pour des raisons politiques ; elles vont également engendrer des polémiques non seulement historiques, mais également politiques, donnant lieu à d'âpres disputes entre historiens de tendances politiques différentes : histoire et politique s'entremêlent ici étroitement.

Notre étude vise à montrer quelle fut la portée, en Russie, des événements de 1905-1907 sur les historiens et leurs recherches. En ce qui concerne le terme « historien », il est utilisé ici dans une acception large. Il comprend tant des historiens ayant reçu une formation historienne à l'université (Vasilij Semevskij (18481916), Pavel Miljukov (1859-1943), Mihail Bogoslovskij (1867-1929), Mitrofan Dovnar-Zapol'skij (1867-1934), Nikolaj Pavlov-Sil'vanskij (1868-1908), Mihail Pokrovskij (1868-1932), Mihail Geršenzon (1869-1925), Aleksandr Presnjakov (1870-1929), Aleksandr Kizevetter (1866-1933) etc.), que des « autodidactes », c'est-à-dire sans formation universitaire, ou alors dans un autre domaine que celui de l'histoire (Vasilij Jakovlev-Bogučarskij (1860-1915), Pavel Ščegolev (18771931), Mihail Lemke (1872-1923), etc.). De plus, même si le domaine des 
recherches historiques en Russie s'institutionnalise fortement à partir de la seconde moitié du XIX ${ }^{\mathrm{e}}$ siècle, il existe toute une lignée historienne hors de l'université. Des « indépendants » comme Pavlov-Sil'vanskij, Semevskij, Geršenzon, ou des « autodidactes » comme Jakovlev-Bogučarskij, Ščegolev ou Lemke, ont, par leurs travaux sur une problématique précise (le féodalisme pour Pavlov-Sil'vanskij, la question paysanne pour Semevskij), par leurs publications de documents et de sources historiques (Lemke, Ščegolev, Jakovlev-Bogučarskij), ou par leurs « essais » historico-philosophiques (Geršenzon), nourri les recherches historiques de manière conséquente. Bien que la production de discours sur le passé, le récit historique, ainsi que les méthodes qui ont servi à l'élaborer soient fort divers chez ces historiens, ils ont tous en commun d'avoir consacré une grande partie de leur vie professionnelle à l'histoire de la Russie.

\section{Un engagement politique renforcé}

Il est difficile d'évaluer l'engagement politique des historiens ${ }^{1}$. Leur politisation n'implique pas nécessairement qu'ils appartiennent à une organisation politique, une telle appartenance n'étant pas le seul mode d'expression de l'engagement politique. Ainsi, Geršenzon ne rejoignit jamais aucune organisation politique ou sociale, mais fut un temps proche du marxisme avant de se tourner vers le libéralisme. Toutefois, si, quantitativement, les historiens ne sont guère nombreux par rapport à l'ensemble de l'intelligentsia professionnelle russe du début du $\mathrm{xx}^{\mathrm{e}}$ siècle, leur participation à la vie sociale et politique russe est importante ${ }^{2}$. De nombreux historiens collaborent activement aux organes périodiques de tendance libérale ou socialiste, et participent aux discussions politiques qui ont lieu dans diverses organisations publiques. Enfin, les historiens jouent un rôle important dans la formation d'organisations politiques. L'exemple le plus connu est celui de Miljukov qui rentre en Russie en avril 1905 et fonde le parti constitutionnel-démocrate (KD) qu'il dirige de 1907 à 1917. Les événements de 1905 renforcent d'ailleurs souvent les historiens déjà politisés dans leurs convictions politiques, qu'ils concrétisent par un engagement au sein des différents partis et organisations apparus il y a peu. Pokrovskij par exemple se rapproche des bolcheviks à la veille de la révolution de 1905 et entre au parti social-démocrate (SD) en avril de la même année. Nikolaj

1. L'historien américain Robert F. Byrnes a montré que si l'impact de 1905 sur Vasilij Ključevskij fut passager, il n'en fut pas moins réel et fortement révélateur de l'importance, de l'espoir, puis de la déception que cet événement suscita chez lui. Robert F. Byrnes, «Kliuchevskii and the revolution of 1905 », in François-Xavier Coquin, Céline Gervais-Francelle, éds., 1905: la première révolution russe (actes du colloque international organisé du 2 au 6 juin 1981), P.: Institut d'études slaves, 1986, p. 79-100. V. A. Murav'ev considère quant à lui que 1905 aurait intensifié la crise dans laquelle l'historiographie «bourgeoise » était plongée durant ces années : V. A. Murav'ev, «Revoljucija 1905-1907 gg. i russkie istoriki (k postanovke problemy)» [La révolution de 1905-1907 et les historiens russes (poser le problème)] in Intelligencija i revoljucija, $X X$ vek [Intelligentsia et révolution, $X X^{\mathrm{e}}$ siècle], M.: Nauka, 1985, p. 68-74.

2. À ce sujet, cf. Murav’ev, « Revoljucija 1905-1907 gg. i russkie istoriki... », p. 69. 
Rožkov (1868-1927), qui s'investit dans la politique dès 1904, entre dans les rangs des bolcheviks après les événements du Dimanche rouge. Aleksej Dživelegov (1875-1952), proche des tendances libérales avant la révolution, entre au parti KD en 1905, devenant un de ses membres les plus actifs, à tel point que, jusqu'en 1908, il met ses occupations scientifiques entre parenthèses. Quant à Semevskij, il est arrêté pour la première fois à la veille du Dimanche rouge ${ }^{3}$. Libéré quelques mois plus tard, il intègre en 1906 l'organe directeur du parti nouvellement créé des Socialistes du peuple (narodnye socialisty), où il ne se fait toutefois guère remarquer. Nikolaj Kareev (1850-1931) entre au parti KD, dont il est un membre très impliqué4. On peut encore citer Kizevetter, pour qui les années 1905-1907 sont synonymes d'une grande activité politique. Entré en octobre 1905 dans le parti KD, il devient membre du comité central en janvier 1906 et est un de ses orateurs les plus dynamiques durant les campagnes de la Première et de la Deuxième Douma. Aleksandr Presnjakov (1870-1929), proche des marxistes «légaux » avant 1905, bascule après les événements du Dimanche rouge dans les rangs des membres actifs du parti KD. Boris Syromjatnikov (1874-1947) se rapproche en 1905 des KD d'abord, puis des Octobristes, tandis que Sergej Mel'gunov (1879-1956) entre au parti KD en 1906 avant de rallier les Socialistes du peuple.

Dès lors, si les événements de 1905 n'ont fait que renforcer certains historiens dans leurs opinions politiques, ils ont pu être déterminants pour d'autres. Cet engagement politique, qu'il soit antérieur à 1905 ou qu'il en soit une conséquence directe, va parfois créer une opposition, une rupture nette entre certains historiens. Ainsi, Pokrovskij, membre du parti SD, prône désormais une vision marxiste de l'histoire ; il engage avec Miljukov et Kizevetter, tous deux membres du parti $\mathrm{KD}^{5}$, des polémiques historiques dans lesquelles l'aspect politique est aisément perceptible.

\section{Nikolaj Pavlov-Sil'vanskij : le choc du Dimanche rouge}

Il est un historien pour qui 1905 a joué un rôle déterminant. Il s'agit de Nikolaj Pavlov-Sil'vanskij, à qui l'on doit les théories les plus importantes sur le

3. Des personnalités intellectuelles (dont Maksim Gorkij) désireuses d'éviter l'affrontement violent qui se prépare, se rendent, le 8 au soir, auprès de Witte, l'enjoignant d'accepter la pétition des ouvriers. Semevskij avait déjà été démis de ses fonctions d'enseignant à l'université de Moscou en 1886, pour ses prises de position critiques envers le gouvernement russe en ce qui concerne la question paysanne.

4. Kareev avait déjà été démis de ses fonctions de professeur à l'université de Saint-Pétersbourg en 1899 pour avoir critiqué les mesures policières prises à l'encontre des étudiants.

5. En ce qui concerne Pokrovskij et Kizevetter, voir la deuxième partie de cette étude. En ce qui concerne les relations de Pokrovskij et de Miljukov, celui-ci a écrit un article sur Pokrovskij, publié en 1937 à Paris dans la revue Sovremennye Zapiski, et dans lequel il revient sur leurs relations. Cf. P. N. Miljukov, «Veličie i padenie M. N. Pokrovskogo (epizod iz istorii nauki v SSSR) » [Grandeur et chute de M. N. Pokrovskij (un épisode de l'histoire de la science en URSS)], in Očerki istorii istoričeskoj nauki [Essais sur l'histoire de la science historique], M. : Nauka, 2002, p. 505-524. 
féodalisme en Russie ${ }^{6}$. Pavel Ščegolev raconte d'ailleurs le choc que le Dimanche rouge a provoqué sur le chercheur :

Les événements du 9 janvier 1905 l'ébranlèrent au plus profond de son âme. Ce jour-là, il errait avec moi dans les rues de Pétersbourg, il vit les attaques de la cavalerie et les fusillades en salves. [...] Nous courûmes à la maison et nous vîmes par la fenêtre les gens se traîner, ensanglantés [...]. Nikolaj Pavlovič était brisé, choqué. Il sanglotait, n'arrivait pas à se reprendre. Il se frappait littéralement la tête contre le mur, et à travers ses larmes, il répétait toujours les mêmes paroles : que font-ils ? que font-ils ? Aux larmes succéda une profonde indignation. ${ }^{7}$

Si, juste après le décret impérial du 12 décembre 1904, Pavlov-Sil’vanskij considérait encore que les réformes dépendaient de «la volonté autocrate de sa majesté impériale $»^{8}$, désormais, rejetant sa foi dans le tsarisme (il évoque les «actes terroristes » d'une autocratie « blessée à mort » $)^{9}$, il participe activement au congrès des zemstvos et à la campagne pour les élections de la Première Douma. Il se rapproche rapidement du parti KD et publie dans le journal Naša žizn' [Notre vie] articles et pamphlets ${ }^{10}$. Dès 1905 , des établissements d'enseignement supérieur « libres » sont créés, qui permettent d'enseigner à des historiens comme PavlovSil'vanskij à qui la carrière universitaire avait été fermée pour diverses raisons ${ }^{11}$. À la lumière des événements de 1905, il critique l'historiographie russe à l'occasion de deux cours qu'il donne en 1906 et 190712 .

Dans « La révolution et l'historiographie russe » (juin 1907), Pavlov-Sil'vanskij analyse les vues des historiens ayant invoqué le développement original de la Russie. C'est Miljukov qui est pointé ici, avec sa théorie du contraste, sa conception de la différence profonde des développements historiques russe et européen. Et

6. N. P. Pavlov-Sil'vanskij, Feodalizm v Rossii [Le Féodalisme en Russie], M.: Nauka, 1988 [SPb, 1907, 1910].

7.P. E. Ščegolev, «Pamjati N.P. Pavlova-Sil`vanskogo » [À la mémoire de N. P. PavlovSil'vanskij], Minuvščie gody, n 10, 1908, p. 309-320.

8. « Notre histoire nous donne la certitude que le puissant Etat russe, aujourd'hui de même que lors des désastreuses années précédentes, sortira régénéré de ces ébranlements externes et internes, en entrant dans la voie de ces grandes réformes qui ont toujours assuré son succès dans les affaires extérieures et ont été un garant important de son développement ininterrompu ». S. N. Valk, «Vstupitel'naja lekcija N. P. Pavlova-Sil'vanskogo » [Conférence inaugurale de Pavlov-Sil'vanskij], in Voprosy istoriografii i istočnikovedenija istorii SSSR, M.-L., 1963, p. 619 .

9. V. A. Murav'ev, «Dve lekcii N.P. Pavlova-Sil'vanskogo ("Istorija i sovremennost”, "Revoljucija i russkaja istoriografija") » [Deux cours de N. P. Pavlov-Sil'vanskij (« Histoire et présent », «La révolution et l'historiographie russe »)], in Istorija i istoriki. Istoriografičeskij ežegodnik, 1972, M., 1973, p. 354.

10. S. V. Čirkov, « N. P. Pavlov-Sil’vanskij i ego knigi o feodalizme» [N. P. PavlovSil'vanskij et ses livres sur le féodalisme], in N. P. Pavlov-Sil'vanskij, Feodalizm v Rossii, p. 606-607.

11. Pour plus de détails, cf. Murav'ev, « Revoljucija 1905-1907 gg. i russkie istoriki... », p. 72, ainsi que Valk, «Vstupitel’naja lekcija... »,p. 618.

12. Ces deux cours ont été publiés : Murav’ev, « Dve lekcii N. P. Pavlova-Sil'vanskogo », p. 337-364. 
c'est précisément en s'appuyant sur les événements de 1905 que Pavlov-Sil'vanskij met à mal cette conception :

Dans notre historiographie jusqu'il y a peu, [...] l'idée de l'originalité absolue du processus historique russe dominait. L'historiographie nous a enseigné que le développement de notre histoire ancienne a eu lieu «à l'envers » du développement de l'histoire occidentale, qu'aucune loi, loi du développement, loi de la vie, ne s'appliquait à elle. Le caractère erroné de ce point de vue a été ébranlé plus fortement que jamais ces trois dernières années, lorsque la réalité, l'histoire, corrigeant les erreurs de l'historiographie, nous a donné des leçons pratiques, nous mettant face à des événements historiques grandioses. Tous, à leur grand étonnement, ont pu voir avec cet exemple que l'histoire russe, peut-être pas dans sa totalité, mais dans certains moments-clés, se développe de façon fort semblable à l'histoire occidentale. Tous sont frappés par la ressemblance de la révolution russe avec les révolutions française et allemande, tous découvrent soudain la méthode comparative, apprennent à percevoir la ressemblance fondamentale des faits sous l'enveloppe trompeuse de la différence extérieure. Ces leçons concrètes de ressemblance ont été inattendues avant tout pour nos historiens, pour ceux qui s'étaient habitués à la doctrine, dominante dans notre historiographie, de la différence dans le processus du développement de l'histoire russe et occidentale. Notre historiographie, prise en masse, n'a de loin pas été à la hauteur des exigences de l'histoire : contribuer à la connaissance de soi du peuple, à la compréhension non seulement du passé, mais aussi du présent et du futur. ${ }^{13}$

Maintenant qu'il est devenu clair pour tout le monde, écrit également PavlovSil’vanskij en février 1906, que

de grands événements ont lieu sous nos yeux, une question doit être posée aux historiens : qu'avons-nous fait, en analysant et en imprimant de vieux actes, en publiant des sources que personne ne lisait à part nous, en travaillant dans les sociétés archéologiques, où personne ne se rendait hormis nous, ainsi que dans les petits auditoires de la faculté d'histoire et des lettres (istoriko-filologičeskij fakul'tet), dans lesquels les étudiants se comptaient en dizaines $?^{14}$

Pavlov-Sil'vanskij ne se contente pas de critiquer avec force l'historiographie russe de son époque, il cherche surtout à élaborer une nouvelle conception de l'histoire de la Russie à la lumière de 1905 . Dans « Histoire et présent ${ }^{15}$, il souligne tout d'abord la similitude de la révolution de 1905 avec la Révolution française. L'histoire se répète, écrit-il, n'en déplaise aux « adversaires des lois sociologiques qui, en regardant deux tableaux peints avec des couleurs différentes, sont incapables de percevoir le même dessin et qui, en raison de la diversité des faits historiques, ne perçoivent pas l'unité du processus historique » ${ }^{16}$. Énumérant les ressem-

13. Murav’ev, « Dve lekcii N. P. Pavlova-Sil’vanskogo », p. 356-357.

14. Valk, «Vstupitel’naja lekcija N. P. Pavlova-Sil’vanskogo »,p. 619.

15. Au sujet de ce premier cours de Pavlov-Sil'vanskij, dispensé le 21 février 1906, cf. Valk, «Vstupitel'naja lekcija N. P. Pavlova-Sil’vanskogo », p. 617-626. 
blances entre ces deux événements, Pavlov-Sil'vanskij termine par une comparaison entre les forces politiques en action ici et là :

les mêmes partis d'avant-garde : les jacobins et les girondins, les uns avec un programme extrême, prônant la république et la violence révolutionnaire ; les autres, les démocrates constitutionnels, reconnaissables à leur modération, leur académisme idéaliste (idealističeskaja akademičnost') et leur manque de caractère pratique, rappellent les girondins. ${ }^{17}$

Pavlov-Sil'vanskij se tourne ensuite vers le passé de la Russie pour rattacher 1905 à toute une tradition révolutionnaire russe. Le «mouvement de libération » ${ }^{18}$ qui a précédé et préparé 1905 a commencé avec Radiščev que l'historien nomme « notre premier révolutionnaire » :

Dans son discours archaïque qui pour la première fois résonne librement de nos jours, cent trois ans après sa mort, on entend le même courage, la même audace qui font la force de notre révolution. Radiščev, les décembristes, les années 1840 et 1860 , les narodovol'cy, les marxistes et les socio-démocrates, les populistes et leurs successeurs les socio-révolutionnaires, telles sont les étapes principales de notre grand mouvement de libération, sans précédent dans l'histoire par le nombre de victimes, par la force de leur héroïque sacrifice de soi. ${ }^{19}$

Ses propos sur la lutte révolutionnaire initiée par Radiščev permettent à PavlovSil'vanskij d'ancrer la filiation révolutionnaire plus loin encore dans le passé. Car pour lui, les sources de ce mouvement de libération, dont l'aboutissement est 1905, se cachent dans la lutte pluriséculaire du peuple russe pour se libérer du joug de l'État. Dans la libération politique actuelle, c'est toute l'histoire ancienne qui se fait entendre : le 9 janvier, les ouvriers ont porté une requête pacifique au tsar, de même que le faisait le peuple au XVII ${ }^{\text {e }}$ siècle. « Notre lutte avec le pouvoir de l'État remonte à l'histoire ancienne, lorsque le droit à la révolte était un fait acquis et constant $»^{20}$ : de Stenka Razin aux révoltes paysannes durant le règne de Paul Ier en passant par les soulèvements cosaques et la révolte de Pugačev, de la lutte obstinée qui opposa les « différentes classes de la société et la société et l'État » ${ }^{21}$ au Temps des Troubles, à la «révolution» de juin 1648 à Moscou, «toute notre histoire ancienne, comme aujourd'hui, est inondée du sang des révoltes populaires $»^{22}$. Cette lutte, «telle une maladie chronique, se cache à l'intérieur, mais parfois de violentes crises éclatent. Au conflit violent succède un armistice, puis l'armistice à

16. Murav’ev, « Dve lekcii N. P. Pavlova-Sil’vanskogo », p. 342.

17. Ibidem.

18. Cette expression, amplement utilisée au début du $\mathrm{Xx}^{\mathrm{e}}$ siècle, englobait tous les types d'oppositions : révoltes paysannes, cercles politiques, écrivains « radicaux », etc.

19. Murav’ev, « Dve lekcii N. P. Pavlova-Sil’vanskogo », p. 343.

20. Ibid., p. 344.

21. Ibid., p. 348.

22. Ibid., p. 344. 
nouveau est brisé par un conflit : la révolution de 1648, après vingt ans d'accalmie, trouve son aboutissement dans la révolte de Razin ${ }^{23}$. D'où l'attention particulière que Pavlov-Sil'vanskij porte aux théories du pouvoir en Russie, à la conception de l'absolutisme - «mythe», voire «mensonge des sources officielles ${ }^{24}-$, apparue en Russie avec Ivan III et demeurée « livresque », « artificielle », « non populaire »; ses fondements ont souvent été mis à mal par la réalité de l'histoire russe, parsemée de révoltes populaires, et par le fait que le peuple ne se représentait pas le pouvoir comme les théoriciens le prétendaient ${ }^{25}$.

Faiblesse de l'autocratie et «force du droit à la révolte », tels sont donc pour Pavlov-Sil'vanskij les deux faits essentiels, perceptibles tout au long de l'histoire russe, et qu'il s'attachera désormais à mettre constamment en avant. Les événements de 1905 s'inscrivent dans cette lutte pluriséculaire de libération du peuple, ils en forment en quelque sorte l'aboutissement.

1905 a donc eu un impact considérable sur l'historien, au niveau politique d'abord, ce que démontre son activité dans l'aile gauche du parti KD après le Dimanche rouge. Or, le politique déborde sur les interprétations historiques de Pavlov-Sil'vanskij qui écrit que «la Grande Révolution russe» l'a poussé à terminer rapidement son ouvrage sur le féodalisme dans la Russie ancienne, publié en $1907^{26}$. Toutefois l'historien ne se contente pas d'évoquer dans ses cours la lutte antiféodale du peuple russe et, sous l'influence des événements politiques, de réinscrire le développement historique russe dans la lignée de celui de l'Occident, refusant encore plus fortement qu'auparavant le particularisme russe ; l'histoire des oppositions politiques, qui intéressait déjà ce spécialiste du féodalisme russe avant 1905, prend désormais dans ses travaux une place primordiale, ce que révèlent d'ailleurs ses archives : tous ses plans de publication pour l'année 1905 comprennent des projets consacrés au décembrisme ${ }^{27}$. Et seule sa mort prématurée en 1908 l'empêchera de mener à bien ses réflexions.

La révolution de 1905 a a influencé d'autres historiens encore : certains recherchent dans le passé de la Russie les analogies avec cet événement, afin de mieux l'appréhender. Ainsi, Mihail Bogoslovskij (1867-1929), spécialiste de l'histoire russe du XVI ${ }^{\mathrm{e}}$ siècle au XVIII ${ }^{\mathrm{e}}$ siècle, publie en 1905 et en 1906 deux brochures dans

23. Ibid., p. 349.

24. Ibid., p. 343.

25. Dans son cours, Pavlov-Sil'vanskij passe en revue l'histoire de cette lutte du peuple pour se libérer, citant un grand nombre de faits visant à montrer la faiblesse de l'autocratie et la force des révoltes populaires.

26. V. A. Murav’ev, «Lekcionnye kursy N.P. Pavlova-Sil'vanskogo v vysših vol'nyh učebnyh zavedenijah Peterburga », Arheografičeskij ežegodnik za 1969 god, M., 1971, p. 250. Cité par S. V. Čirkov, « N. P. Pavlov-Sil'vanskij i ego knigi ... », p. 624.

27. RGIA (Rossijskij gosudarstvennyj istoričeskij arhiv - Archives d'histoire de l'État de Russie), f. 1014, op. 1, d. 67,1. 33,38. Cité in G. A. Nevelev, Dekabristy i dekabristovedy [Les Décembristes et les spécialistes du décembrisme], SPb. : Tehnologos, 2003, p. 265. 
lesquelles il analyse les précédents à la révolution de $1905^{28}$. Dans ces deux petits textes, Bogoslovskij montre qu'en Russie, les mouvements ou actes de type constitutionnel ont toujours échoué. Prenant souvent en exemple les mêmes événements que Pavlov-Sil'vanskij, Bogoslovskij, historien plutôt conservateur, opposé aux révolutions et aux changements violents ${ }^{29}$, en tire des conclusions opposées : il montre la force de l'autocratie, la faiblesse des mouvements d'opposition, la profonde inertie des masses et, enfin, la proximité de la conception théorique du pouvoir divin avec les vues populaires.

Bogoslovskij reviendra toutefois rapidement à ses thématiques précédentes. En effet, c'est surtout sur les historiens favorables à 1905 et proches des mouvements libéraux ou radicaux que l'impact de 1905 est le plus perceptible, et surtout le plus profond. Fortement influencés par les événements révolutionnaires et ayant la possibilité de consulter des archives et des matériaux auparavant inaccessibles, ils vont désormais privilégier les recherches sur les oppositions au tsarisme, qui donneront rapidement lieu à une production historique florissante.

\section{L'histoire du mouvement de libération russe : l'émergence d'une nouvelle historiographie}

Vers la fin du XIX ${ }^{e}$ siècle déjà, les mouvements sociaux en Russie étaient l'objet d'études menées par des historiens tels qu'Aleksandr Pypin (1833-1904), V. Semevskij et V. Jakovlev-Bogučarskij. Toutefois, cette thématique était fortement censurée et les sources en grande partie inaccessibles. Comme le souligne Kizevetter en 1908, très rarement et à titre d'exception, « quelques élus, des écrivains de toute confiance, avaient accès à ces lieux tenus secrets. On leur montrait des documents, ce qui ne signifiait pas du tout qu'ils obtiendraient le droit d'utiliser ce qu'ils voyaient pour leurs publications $»^{30}$. En conséquence, lorsque des informations sur les acteurs du mouvement de libération circulaient, les faits étaient souvent déformés. Dès 1905, les verrous des portes des archives de l'État commencent à sauter et des historiens obtiennent l'autorisation d'y travailler. D'une façon plus générale, c'est l'histoire intellectuelle sociale, culturelle et politique du XIX siècle qui devient accessible aux chercheurs. Kizevetter s'en réjouit et salue, en 1908, la première publication collective sur l'histoire de la Russie au $\mathrm{XIX}^{\mathrm{e}}$ siècle $^{31}$.

28. M. M. Bogoslovskij, Iz istorii verhovnoj vlasti v Rossii [À propos de l'histoire du pouvoir suprême en Russie], M., 1905, et Konstitucionnoe dviženie 1730 g. [Le Mouvement constitutionnel de 1730], M., 1906.

29. T. I. Halina, « Mihail Mihajlovič Bogoslovskij (1867-1929) », in A. A. Černobaev, réd., Istoriki Rossii : biografii, M. : Rosspen, 2001, p. 426-433.

30. A. Kizevetter, « Carstvovanie Aleksandra I v novom osveščenii » [Le règne d'Alexandre Irer : nouvel éclairage], Russkaja mysl', 1908, p. 99-100.

31. Ibid., p. 99-118. Il s'agit de Istorija Rossii v XIX v. [L'Histoire de la Russie au XIX siècle], M.: Granat, 1907. 
Fait capital, la censure préalable à l'impression est supprimée en novembre 1905. Même si la censure ne disparaît pas, puisqu'elle s'applique désormais aux ouvrages imprimés, ce qui signifie que les textes peuvent être confisqués et détruits après leur publication, l'impact de ce décret impérial est immédiat, et immense. Dès la fin de 1905, un nombre considérable d'ouvrages sur des sujets auparavant censurés est publié (documents, mémoires, correspondance, biographies, etc.). C'est en 1905 par exemple que paraît la première édition complète du pamphlet d'Aleksandr Radiščev, Voyage de Pétersbourg à Moscou, sous la rédaction de Pavlov-Sil'vanskij et de Ščegolev, qui obtiennent le droit d'étudier les archives de l' «affaire » Radiščev $^{32}$. Cette publication, qui rend pour la première fois Radiščev accessible au grand public, est unanimement saluée par la presse libérale et radicale de l'époque ${ }^{33}$. La première étude d'envergure sur Petraševskij et les Petraševcy est due à Semevskij, qui travaille sur cette thématique de 1909 jusqu'à sa mort en $1916^{34}$. On peut également citer les travaux de Geršenzon sur Čaadaev (Geršenzon est le premier à lui avoir consacré une étude ${ }^{35}$ ), Gercen, Ogarev et les décembristes. Ses recherches, bien qu'elles soient antérieures à 1905, sont publiées entre 1906 et $1914^{36}$.

Enfin, pour la première fois, une revue consacrée au mouvement de libération russe est publiée en Russie de façon légale. En janvier 1906, la revue Le Passé (Byloe), éditée de 1900 à 1904 à Londres par Vladimir Burcev (1862-1942), commence à paraître à Saint-Pétersbourg ${ }^{37}$. Le Passé rassemble toutes sortes de documents sur le mouvement de libération russe. Ceux-ci concernent principalement La Volonté du peuple, mais aussi les décembristes, auxquels la revue

32. A. N. Radiščev, Putešestvie iz Peterburga v Moskvu [Voyage de Pétersbourg à Moscou], sous la rédaction de N. P. Pavlov-Sil'vanskij et P. E. Ščegolev, SPb.: Obščestvennaja pol’za, 1905. Pour cette édition, Pavlov-Sil'vanskij écrit une biographie de Radiščev et Ščegolev un article sur le destin de son manuscrit. En 1906, cinq rééditions verront le jour et, en 1907, trois. Les publications se suivront ensuite à un rythme assez rapide. Ščegolev écrira encore un texte sur Radiščev («Iz istorii žurnal'noj dejatel'nosti A. N. Radiščeva, 1789 g. » [L'activité de A. N. Radiščev dans la presse en 1789], Minuvšie gody, n 12, 1908) et participera à la publication de la première édition complète des œuvres de Radiščev en deux tomes en 1907-1908.

33. Par exemple D. Ovsjaniko-Kulikovskij, «Dva slova o Radiščeve » [Deux mots sur Radiščev], Poljarnaja zvezda, n 1,15 décembre 1905.

34. V. Semevskij, M. B. Butaševič-Petraševskij i Petraševcy [M. V. Butaševič-Petraševskij et les Petraševcy], M.: Zadruga, 1922. Des parties avaient déjà été publiées auparavant dans diverses revues, en particulier dans Golos minuv̌̌ego.

35. P. A. Čaadaev, Žizn’i myšlenie [P. A. Čaadaev, Vie et pensée], M., 1908.

36. M. Geršenzon, Social'no-političeskie vzgljady A. I. Gercena [Les opinions sociales et politiques de A. I. Gercen], M., 1906 ; Istorija molodoj Rossii [Histoire de la jeune Russie], M., 1908 ; Dekabrist Krivcov i ego brat’ja [Le Décembriste Krivcov et ses frères], M., 1914. Les travaux de Geršenzon, dont les conceptions s'éloignent assez fondamentalement de celles des autres historiens «de gauche », seront soumis aux critiques de Kizevetter (Russkaja mysl", $\mathrm{n}^{\circ}$ 6, 1908, p. 123-125), Ščegolev (Minuvšie gody, n 1, janvier 1908, p. 300-301) et Semevskij (Golos minuv̌̌ego, n 6, 1914, p. 295-298).

37. Au sujet de la revue Byloe, voir F. M. Lur'e, Hraniteli prošlogo. Žurnal « Byloe » : istorija, redaktory, izdateli [Les conservateurs du passé. La revue «Byloe»: histoire, rédacteurs, éditeurs], L.: Lenizdat, 1990, ainsi que Korine Amacher, « La revue Ce qui fut (1906-1907) : l'émergence d'une historiographie du mouvement révolutionnaire en Russie », in Les sites de la mémoire russe, sous la direction de Georges Nivat, P.: Fayard, tome 2 (à paraître). 
consacre un nombre considérable de travaux, les émigrés politiques tels Gercen, Ogarev ou Bakunin, les « radicaux » et les révolutionnaires des années 1860, différents cercles et groupes socialistes, les socialistes-révolutionnaires, la social-démocratie en Russie, etc. En deux années, un grand nombre de documents il y a encore peu hautement secrets y sont publiés, notamment ceux sur les décembristes, dont la majorité des écrits est enfin accessible au public russe grâce à l'ouverture partielle des archives. La revue connaît un succès immense et son tirage est très élevé : environ 30000 exemplaires, chiffre remarquable pour l'époque si on le compare, par exemple, avec le nombre d'abonnés à la revue Russkaja mysl' [La Pensée russe], qui se situe entre 10000 et 15 000. Le Passé devient rapidement la revue qui publie les sources, les matériaux, les documents, les souvenirs des anciens révolutionnaires des années 1870-1880, libérés de la forteresse de Schlusselbourg à la faveur de 1905. Dans la presse libérale et radicale de l'époque, sa naissance est évoquée en des termes enthousiastes :

Jusqu'il y a peu, jusqu'à cette liberté relative dont la presse bénéficie actuellement et que le gouvernement, malgré toute la cruauté des répressions, n'est dorénavant plus en mesure d'écraser, l'histoire de notre mouvement de libération restait un fruit défendu pour la société russe. Dans ce système où toute vie libre était annihilée, l'étouffement de la mémoire de l'histoire de la société russe était un maillon nécessaire. [...] Ceux qui chérissaient les traditions et qui, instinctivement, se protégeaient de cette mutilation spirituelle, étaient contraints de se nourrir de légendes et de rumeurs orales sur le passé et non d'une véritable connaissance de la vérité historique. [...] Aujourd'hui, parmi les droits que le peuple, dans une certaine mesure, a déjà conquis, le droit à la connaissance véridique du passé de la vie sociale russe [russkaja obščestvennost'] est également chose conquise. Satisfaire cette exigence nationale de connaissance de soi par la compréhension de l'histoire du mouvement de libération nationale, tel est le but de la revue Le Passé. 38

Ainsi, la naissance de la revue symboliserait une nouvelle liberté d'expression en ce qui concerne le passé russe. Après des années de censure vis-à-vis des mouvements d'opposition politique au tsarisme, Le Passé témoigne de la possibilité de se réapproprier l'histoire «nationale», de saisir la «vérité historique », opposée au mensonge de l'historiographie officielle.

Suppression de la censure préalable à l'impression, accès à des archives auparavant interdites, atmosphère « révolutionnaire », changements politiques et sociaux, telles sont, brossées en quelques lignes, les circonstances qui permettent de mieux comprendre la réorientation des recherches historiques vers une thématique auparavant censurée : le «mouvement de libération russe ». Or dans ce champ thématique, qui englobe de nombreux acteurs et mouvements, le décembrisme est au centre. Et comme nous allons le voir, les décembristes sont, dès 1905, mêlés à des polémiques qui dépassent le cadre historique pour toucher le politique.

38. C. Frank, « Novyj istoričeskij žurnal » [Une nouvelle revue historique], Poljarnaja zvezda, $\mathrm{n}^{\circ} 10,17$ février 1906, p. 738-739. 


\section{Les décembristes ou le combat pour la liberté}

Jusqu'en 1905, les historiens qui avaient eu accès aux archives des décembristes étaient toutes des personnes de confiance du régime tsariste ${ }^{39}$. Désormais, les portes des archives de l'État s'entrouvrent pour les chercheurs qui en font la demande, indépendamment de leurs convictions politiques, puisque ceux qui travailleront après 1905 sur cette thématique sont plutôt des historiens « de gauche », qui n'ont en général pas une fonction académique ni un enseignement stable. Enfin, certains sont venus à l'étude de l'histoire du mouvement révolutionnaire russe suite à leur engagement politique. Pour des raisons politiques évidentes d'ailleurs, les recherches historiques consacrées à cette thématique se heurtent à l'hostilité du pouvoir tsariste et restent peu présentes dans les milieux académiques jusqu'en 1917. P. Ščegolev est un des premiers, en mai 1905, à obtenir le droit du ministère des Affaires intérieures d'étudier les documents conservés dans les archives de la Troisième section. Pour la première fois, il est possible d'étudier les idéaux socio-politiques des décembristes sur la base non pas des souvenirs de ceux qui furent libérés après la mort de Nicolas ${ }^{e r}$, de quelques archives privées ou des matériaux parfois publiés à l'étranger ${ }^{40}$, mais sur la base de la masse impressionnante de matériaux conservés aux archives de l'État. Et surtout, à la différence des quelques historiens qui avaient eu accès par le passé aux archives des décembristes, après 1905, les historiens proposent désormais de nouvelles interprétations du décembrisme.

Très rapidement, les écrits, les programmes politiques des décembristes sont publiés ${ }^{41}$, les références, les allusions à la révolte de 1825 se multiplient. Des revues se créent, qui se réclament des valeurs du décembrisme et dans lesquelles les événements de 1905 sont présentés comme l'aboutissement d'une lutte initiée en 1825. La revue Poljarnaja zvezda [L'Étoile polaire], dont le nom est le même que celui de la revue du poète Kondratij Ryleev, un des cinq décembristes pendus après la révolte de 1825 , puis de celle de Gercen à Londres, en est un exemple emblématique $^{42}$. Dans le premier numéro (15 décembre 1905), la rédaction annonce claire-

39. Le premier à avoir eu accès à une partie des documents concernant les décembristes est M. I. Bogdanovič, (Istorija carstvovanija imperatora Aleksandra I i Rossija v ego vremja [L'Histoire du règne de l'empereur Alexandre Ir et la Russie de son temps], SPb., 1869-1871, t. 1-6). Puis on peut mentionner N. K. Šil'der (Imperator Aleksandr I, ego žizn'i i carstvovanie [L'Empereur Alexandre Irer, sa vie et son règne], SPb, 1897-1898, t. 1-4) et N. F. Dubrovin auteur de nombreux articles sur l'époque d'Alexandre Ier (Russkaja žizn' v načale XIX veka [La vie russe au début du XIXe siècle]), parus de 1898 à 1904 dans Russkaja starina.

40. En particulier par Gercen à Londres.

41. Durant ces années, un intense travail de rassemblement et de publications de documents est réalisé. Il est parfois effectué dans l'urgence, en raison des circonstances politiques particulières dans lesquelles se trouve la Russie. Contentons-nous de citer ici un exemple parmi d'innombrables autres : Iz pisem i pokazanij dekabristov. Kritika sovremennago sostojanija Rossii i plany buduščego ustrojstva [Lettres et dépositions des décembristes. Critique de l'état actuel de la Russie et plans d'organisation future], sous la rédaction de A. K. Borozdin, SPb., 1906.

42. Le rédacteur de cette revue est Petr Struve. 14 numéros paraîtront, du 15 décembre 1905 au 19 mars 1906. 
ment son programme : « inculquer dans les esprits et incarner dans les faits » les « grandes traditions révolutionnaires et culturelles », reliées aux noms de Ryleev et de Gercen. Ces traditions «sont plus que des souvenirs historiques. Ils sont une vérité vivante ». Deux articles sont consacrés aux décembristes. Le premier, de Presnjakov, décrit le mouvement décembriste comme le «berceau du développement historique de la pensée sociale russe» et appelle les décembristes les « premiers combattants pour la liberté politique de la Russie » (le terme « pour la première fois » apparaît une dizaine de fois en conclusion de l'article $)^{43}$. Le second article, de Fedor Rodičev ${ }^{44}$, intitulé « 14 décembre 1825 - 14 décembre 1905 », écrit que «de légende et de tradition, l'histoire de la libération russe devient document », allusion à l'ouverture des archives et à l'accès, pour les historiens, aux documents. Les dates du 14 décembre 1825, du 19 février 1861 et du 17 octobre 1905 sont érigées en nouvelles dates nationales, et les décembristes sont décrits comme des martyrs, des hommes courageux, purs et pleins d'abnégation. Le résultat de la révolte était insignifiant, écrit Rodičev, mais le sacrifice n'a pas été vain, Gercen a maintenu vivante la flamme. Aujourd'hui, le gouffre qui sépare le peuple de la société cultivée disparaît, et les premiers à avoir contribué à sa disparition sont les décembristes. La Russie qui se libère en ce début de $\mathrm{XX}^{\mathrm{e}}$ siècle doit se souvenir de sa « première phalange de libération, de ses saints et de ses martyrs $»^{45}$.

Nombreux sont ceux qui, bien que travaillant sur des sujets fort éloignés, se mettent à écrire des articles dans lesquels ils analysent les idéaux socio-politiques des décembristes ${ }^{46}$, témoignant ainsi de l'intérêt profond que cette thématique suscite durant ces années. Cependant, les premiers historiens à se spécialiser dans l'étude du décembrisme sont V. Semevskij, N. Pavlov-Sil'vanskij, P. Ščegolev et M. Dovnar-Zapol'skij. Certains avaient abordé ce sujet avant 1905. Dès 1903, Semevskij avait reçu l'autorisation de consulter les archives secrètes de l'affaire des décembristes (ainsi que de celle des Petraševcy). Quant à Pavlov-Sil'vanskij, il s'agit d'un cas un peu particulier puisque, employé aux Archives de l'État, il eut plus facilement que d'autres accès aux matériaux du mouvement révolutionnaire, ceux de Pestel $^{\prime}$ en particulier, dont il écrit la première biographie scientifique en 1902 ${ }^{47}$. En ce qui concerne Dovnar-Zapol'skij, ses travaux sur le décembrisme, publiés en 1906 et en 1907, représentent une réorientation radicale de ses

43. A. Presnjakov, « Dekabristy » [Les Décembristes], Poljarnaja zvezda, $\mathrm{n}^{\circ} 1,15$ décembre 1905, p. 43-57.

44. F. Rodičev, «14 Dekabrja 1825-14 Dekabrja 1905 g. » [14 Décembre 1825-14 Décembre 1905 », Poljarnaja zvezda, n 1, 15 décembre 1905, p. 81-85. Homme politique et juriste, Fedor Rodičev (1853-1932) est un des fondateurs du parti KD, membre de son comité central. De mars à mai 1917, il est ministre du gouvernement provisoire pour les affaires de la Finlande. Il émigre en 1919.

45. Rodičev, « 14 Dekabrja $1825 \ldots$.. p. 85.

46. Ainsi par exemple Maksim Kovalevskij, qui analyse la «Loi russe » de Pestel’, publiée pour la première fois en 1906 par Ščegolev. M. Kovalevskij, «Russkaja Pravda Pestelja » [ « La Loi russe de Pestel’ »], Minuvšie gody, n 1, janvier 1908.

47. N. P. Pavlov-Sil’vanskij, «P. I. Pestel'», Russkij biografičeskij slovar’ [Le Dictionnaire biographique russe], t. 13, SPb., 1902. 
recherches, puisque son champ d'étude principal est l'histoire de la Lituanie et de la Biélorussie. Enfin, comme il l'écrit lui-même, ce sont les événements de l'année 1905 qui donnent à Ščegolev une nouvelle «impulsion » à son étude du mouvement de libération russe ${ }^{48}$. L'essentiel de ses écrits consacrés au décembrisme ont d'ailleurs été produits entre 1905 et 1917 . Après cette date, il se contentera de les rééditer ${ }^{49}$.

Parmi ces historiens, deux d'entre eux (Semevskij et Dovnar-Zapol'skij) étudient le mouvement décembriste dans son ensemble ${ }^{50}$, les autres se penchant plutôt sur des personnages particuliers. Hormis un long article ${ }^{51}$ dans lequel il analyse les influences des décembristes, Pavlov-Sil'vanskij s'intéresse surtout à Pestel' . Ščegolev, lui, étudie des personnages moins connus et aux destins «tragiques »: Vladimir Raevskij, le « premier décembriste », arrêté dès 1822 et lourdement condamné alors qu'aucune preuve ne fut avancée contre lui, Fedor Šahovskoj, mort fou en 1829, et Petr Kahovskij, tragique et solitaire, incompris et exalté, assassin de Miloradovič et « mal-aimé » des autres décembristes, l'incarnation pour cet historien du révolutionnaire romantique des années 1820 . Pavel Ščegolev est celui qui insiste le plus sur le pathos, l'aspect tragique et l'exaltation révolutionnaire de ces hommes. Ces travaux sont d'ailleurs empreints d'une force évocatrice absente chez les autres historiens, venant probablement du fait que leur auteur est avant tout un spécialiste de la littérature (de Puškin tout particulièrement, auquel il a consacré de nombreux travaux $)^{52}$.

Toutefois, pour la majorité de ceux qui traitent, de près ou de loin, de cette thématique, les décembristes sont les incarnations des héros romantiques et tragiques. Leur courage est souligné de même que leur abnégation, leur sens du sacrifice, leur grandeur, en regard de l'arbitraire et la cruauté du tsarisme. Les décembristes, véritables exemples pour leurs successeurs ${ }^{53}$, sont les premiers hommes à avoir lutté pour la liberté politique en Russie, les premiers à avoir posé clairement la question des droits

48. Cité in Lur'e, Hraniteli prošlogo..., p. 12.

49. P. E. Ščegolev, Dekabristy [Les Décembristes], M.-L., 1926.

50. V. I. Semevskij, Političeskie i obščestvennye idei dekabristov [Les Idées politiques et sociales des décembristes], SPb, 1909 ; M. V. Dovnar-Zapol'skij, Tajnoe ob̌̌čestvo dekabristov [La Société secrète des décembristes], M., 1906; Memuary dekabristov (zapiski, pis'ma, pokazanija, proekty konstitucii, izvlečennye iz sledstvennogo dela, s vvodnoj stat'ej) [Les Mémoires des décembristes (notes, lettres, dépositions, projets de constitution, extraits du dossier d'instruction, avec une introduction)], Kiev, 1906 ; Idealy dekabristov [Les Idéaux des décembristes], M., 1907.

51. N. P. Pavlov-Sil'vanskij, « Materialisty 20-h godov » [Les Matérialistes des années vingt],

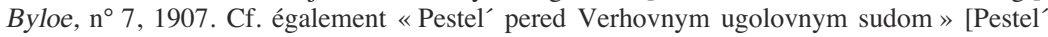
devant la Cour criminelle suprême], Byloe, n² 2-3-4-5, 1906.

52. P. Ščegolev, «Imperator Nikolaj I - tjuremščik dekabristov » [L'Empereur Nicolas Ire, geôlier des décembristes], Byloe, n 5, 1906 ; «Vladimir Raevskij », Vestnik Evropy, avril 1903 ; «Katehizis S. I. Murav’eva-Apostola» [Le Catéchisme de S. I. Murav’ev-Apostol], Minuvšie gody, n 11, 1908 ; «Dekabrist Knjaz’ F. P. Šahovskoj » [Le Prince F. P. Šahovskoj, décembriste], Byloe, n 8, 1907 ; «Ženy dekabristov» [Les Femmes des décembristes], K svetu, SPb, 1904 ; «Petr Grigor’evič Kahovskij. Istoričesko-psihologičeskij etjud » [Petr Grigor'evič Kahovskij. Étude historico-psychologique], Byloe, nº 1-2, 1906.

53. Semevskij, Političeskie i obščestvennye idei dekabristov, p. 602 et 647. 
du peuple, les premiers à avoir «prononcé une sentence contre leur époque, dont la légitimité ne peut pas être remise en doute par l'historien ${ }^{54}$. Gercen, de même que tous les « combattants » de la liberté au XIXe siècle sont les héritiers des décembristes. Ils sont le point de départ, le berceau du mouvement social et de libération russe, même si N. Pavlov-Sil'vanskij relie leur lutte à celle, pluriséculaire du peuple russe contre le pouvoir autocratique. Dans «Les matérialistes des années vingt », l'historien montre la proximité intellectuelle et spirituelle entre les «révolutionnaires matérialistes » des années 1820 et les «révolutionnaires nihilistes » des années $1860^{55}$. Enfin, tous perçoivent la révolution de 1905 comme l'accomplissement de la lutte des décembristes. Ainsi que l'écrit M. Dovnar-Zapol'skij, il aura fallu presque un siècle pour que la société s'éveille, mais les décembristes avaient raison et 1905 en est la démonstration ${ }^{56}$.

Si l'interprétation du décembrisme est, dans ses grandes lignes, commune à tous ces historiens ${ }^{57}$, et si les critiques qu'ils s'adressent restent généralement dans le cadre du domaine scientifique ${ }^{58}$, entre les camps des historiens d'orientation non marxiste et marxiste, les polémiques sont en revanche acerbes.

Ainsi Pokrovskij interprète le décembrisme fort différemment de tous les historiens dont il a été question jusqu'à présent. Dans L'Histoire de la Russie au $X I X^{\mathrm{e}}$ siècle, ouvrage collectif publié en 1907, Pokrovskij est l'auteur du chapitre consacré à l'idéologie des décembristes. Les décembristes, affirme-t-il, ne furent pas des révolutionnaires désireux de changer l'ordre social, mais des hommes qui visaient avant tout à satisfaire leurs intérêts de caste militaire et nobiliaire. Cette interprétation, fondée sur une analyse de classe, n'est pas du goût de Kizevetter, qui déconstruit dans une recension les analyses marxistes et les interprétations de Pokrovksij. Mais c'est surtout le ton « désagréable », dénué de respect, avec lequel Pokrovskij parle des «pionniers du mouvement de libération russe » qui irrite le

54. Dovnar-Zapol'skij, Idealy dekabristov, p. 422.

55. Pavlov-Sil'vanskij, « Materialisty 20-h godov », p. 123.

56. Dovnar-Zapol’skij, Idealy dekabristov, p. 245.

57. Jusqu'à la fin des années 1950, on s'intéresse peu aux travaux « pionniers » de ces historiens sur le décembrisme, si ce n'est pour les critiquer. Un changement a lieu dès les années 1960 . On « redécouvre » ces historiens, et, pour ne citer qu'un exemple, un historien consacre coup sur coup plusieurs articles à leurs études sur les décembristes: G. A. Nevelev, « N. P. PavlovSil’vanskij - istorik dekabristov » [N.P. Pavlov-Sil'vanskij - historien des décembristes] Osvoboditel'noe dviženie v Rossii, Vyp. 1, Saratov, 1971, p. 53-69; G. A. Nevelev, «Istorija dekabristov v trudah Semevskogo » [L'Histoire des décembristes dans les travaux de Semevskij], Istorija i istoriki. Istoriografija istorii SSSR. Sbornik statej, M., 1973, p. 232-257 ; G. A. Nevelev, «Issledovanija o dekabristah M. V. Dovnar-Zapol’skogo » [Les travaux sur les décembristes de M. V. Dovnar-Zapol'skij], Vestnik Leningradskogo universiteta. Istorija, jazyk, literatura, Vyp. 1, n² 2, janvier 1971, p. 53-56. Les travaux de G. A. Nevelev sur ce sujet ont été réédités en 2003 dans G. A. Nevelev, Dekabristy i dekabristovedy, op.cit.

58. Ainsi par exemple, Pavlov-Sil'vanskij est l'auteur d'une recension sur un ouvrage de Dovnar-Zapol'skij, à qui il reproche toute une série d'erreurs factuelles et un manque de rigueur méthodologique, due à un travail effectué trop vite. N.P. Pavlov-Sil'vanskij, «M. V. Dovnar-Zapol'skij. Tajnoe obščestvo dekabristov» [M. V. Dovnar'-Zapol'skij. La Société secrète des décembristes], Byloe, n 5, 1907, p. 312-319. 
plus Kizevetter ${ }^{59}$. Derrière les interprétations de l'historien marxiste, ce sont, pour Kizevetter, les polémiques politiques de l'époque actuelle qui émergent :

Dans la représentation historique du passé s'immiscent des thèmes et des slogans actuels purement partisans. La science historique se transforme en serviteur de la politique. [...] Sous couvert de caractère scientifique, ces pages se font l'écho d'une lutte née non pas dans les murs du bureau d'un chercheur, mais dans une arène fort différente. En la personne des décembristes, ce sont la modération, l'inconsistance et l'esprit antidémocratique de la « bourgeoisie libérale » que ces auteurs stigmatisent. Sous le masque d'études historiques une polémique que l'on pourrait entendre dans un meeting se fait jour. Mais est-il «scientifique » de rendre responsable la jeunesse noble du début du XIX ${ }^{\mathrm{e}}$ siècle des « péchés » de la bourgeoisie libérale du début du XXe siècle $?^{60}$

En fait, ironise Kizevetter, ce que Pokrovskij reproche aux décembristes, c'est de n'avoir pas été des « socio-démocrates bolcheviques ${ }^{61}$. Suite à plusieurs recensions critiques d'historiens, Pokrovskij dénonce une véritable « lutte de classe par écrit?»(klassovaja bor'ba na per'jah), et dans cette unanimité des critiques, il perçoit, « perçant sous le tas de partis différents », un « relent de classe bourgeois ». « Ne divisons pas les historiens en bourgeois et en marxistes, lui répond alors Kizevetter, mais en historiens sérieux et pas sérieux, et reconnaissons qu'on trouve les uns et les autres dans des partis politiques fort différents ${ }^{62}$.

Les décembristes se retrouvent donc dès 1905 mêlés à une polémique qui dépasse les questions historiographiques pour contaminer le politique, au point de provoquer une rupture nette qui ne fera d'ailleurs que s'intensifier chez certains historiens. Cette polémique dépassera d'ailleurs le cadre de ces années, comme le montre en 1913 une recension très critique de Vasilij Semevskij sur les chapitres de l'ouvrage de Pokrovskij L'Histoire russe depuis les temps les plus anciens consacrés aux décembristes ${ }^{63}$. À nouveau, c'est l'analyse de classe du mouvement décembriste qui est au centre de la critique de Semevskij, ainsi que la vision de la révolte décembriste comme une révolte de palais (dvorcovyj perevorot) de plus, dans la lignée de celles que la Russie connut durant le XVIII ${ }^{\mathrm{e}}$ siècle ${ }^{64}$. Mais la lecture

59. Kizevetter, « Carstvovanie Aleksandra I v novom osveščenii », p. 113.

60. Ibid., p. 103.

61. Ibid., p. 114.

62. A. Kizevetter, «Dva slova M. N. Pokrovskomu» [Deux mots à M. N. Pokrovskij], Russkaja mysl’, n 5, mai, 1908, p. 204-208.

63. M. N. Pokrovskij, Russkaja istorija s drevnejših vremen [L'Histoire russe depuis les temps les plus anciens], M., 1910-1913,t. 1-5.

64. «Lorsque l'école juridique dominait dans la science historique, on ne s'intéressait pas aux phénomènes économiques comme cela aurait dû être le cas. Nous avons maintenant un grand nombre d'excellents travaux sur l'histoire économique de la Russie. Leurs auteurs [...] bien qu'ils accordent une importance très grande aux causes économiques, ne veulent pas expliquer tous les phénomènes historiques par l'économie et ne sont pas d'accord, par exemple, de soutenir que le mouvement décembriste est déterminé par la montée des prix du blé ». Cette recension de V. Semevskij sur l'ouvrage de Pokrovskij a paru dans Golos minuvšego, n 7 , 1913 , p. $250-254$. 
du décembrisme oscillera encore longtemps entre diverses interprétations et, après 1917, Pokrovskij lui-même réorientera plusieurs fois ses théories sur le décembrisme ${ }^{65}$, jusqu'à ce que la « conception » de Lenin du décembrisme, formulée par l'historienne soviétique Milica Nečkina après la mise au ban de l'école de Pokrovskij en 1936, ne devienne la seule valable pendant de nombreuses années ${ }^{66}$. Nečkina reprochera alors aux historiens «bourgeois » de n'avoir pas suivi les « indications » de Lenin et de n'avoir perçu le phénomène décembriste que du point de vue des idées constitutionnelles et des conditions politiques du règne d'Alexandre Ier67.

On peut néanmoins se demander si les textes dans lesquels Lenin parle des décembristes étaient, comme le suggère Nečkina, tous connus dans le milieu historique, non marxiste qui plus est ${ }^{68}$. C'est Nečkina qui édifiera plus tard, sur la base des quelques écrits de Lenin sur cette question, une interprétation qui deviendra « officielle » dès la fin des années 1930 en Union soviétique. Toutefois, en encensant les décembristes, en les dépeignant comme des héros tragiques, comme les premiers révolutionnaires d'une longue chaîne qui se conclut par 1905, ces historiens contribuent au renforcement $\mathrm{du}$ 《mythe » décembriste comme «pères » $\mathrm{du}$ mouvement de libération russe. Ce mythe, initié par Gercen et que Plehanov et Lenin se réapproprient à leur manière dès $1900^{69}$, trouve en quelque sorte une légitimité scientifique dans de nombreux travaux d'historiens témoins de la révolution de 1905. Des historiens qui, en réinscrivant cet événement dans un développement

65. Au sujet des analyses de Pokrovskij sur le décembrisme, voir par exemple Gino Sitran, «L'historiographie du mouvement décembriste dans les cinquante dernières années », in Le 14 décembre 1825. Origine et héritage du mouvement des décembristes (textes recueillis par Alexandre Bourmeyster), P.: Institut d'études slaves, 1980, p. 145-150.

66. M. V. Nečkina, Dviženie dekabristov [Le Mouvement des décembristes], M. : Izd. AN SSSR, 1955. Cf. en particulier le premier chapitre, intitulé « Dekabristy kak issledovatel'skaja problema » [Les Décembristes comme thème de recherche] (p. 5-49). Nečkina y analyse les textes dans lesquels Lenin évoque les décembristes.

67. Nečkina, Dviženie dekabristov, p. 29 et ss.

68. «Bien que les thèses de Lenin sur le mouvement des décembristes fussent connues durant la période pré-révolutionnaire presque dans leur totalité, la science historique ne les a pas immédiatement intégrées et appliquées. Le tableau pré-révolutionnaire de l'étude des décembristes restait complexe et contradictoire. Une lutte de classe aiguë imprégnait l'historiographie décembriste ». Nečkina, Dviženie dekabristov, p. 29.

69. Plehanov est l'auteur d'un discours prononcé à Genève en 1900 pour le $75^{\mathrm{e}}$ anniversaire de la révolte des décembristes. G. Plehanov, 14-e dekabrja 1825 goda [Le 14 décembre 1825], M., O. N. Rutenberg, 1926. Dans ce discours, Plehanov, qui s'inscrit dans la lignée de la «tradition » de Gercen (un discours dénué de toute analyse de classe, comme le soulignera Nečkina dans Dviženie dekabristov (p. 19)), décrit les décembristes comme des héros, des « martyrs » décidés à marcher au devant de la mort pour le « bien de la patrie », persuadés que leur mort « réveillera » les Russes « endormis », résolus à montrer par leur sacrifice la voie aux génération futures. Telle serait, pour Plehanov, la raison pour laquelle les décembristes ne se seraient pas vraiment défendus sur la place du Sénat (G. Plehanov, 14-e dekabrja 1825 goda, p. 3-24). Rappelons aussi l'épigraphe que Lenin choisit en 1900 pour le journal marxiste russe Iskra — « de l'étincelle jaillira la flamme » ( « iz iskry vosgoritsja plamja ») —, vers du poème du décembriste Odoevskij en réponse à l' «épître en Sibérie » (Poslanie v Sibir') de Puškin, ainsi que ses articles divers, en particulier « Pamjati Gercena » [Â la mémoire de Gercen], daté de 1912 . 
historique normal, en lui donnant la valeur d'une loi historique, s'intègrent également (eux, les historiens de tendance « libérale » ${ }^{70}$ ), à cette chaîne, dont 1905 représente l'aboutissement logique et, surtout, victorieux. Car dans les écrits des années 1906-1907 de la frange historienne libérale perce non seulement l'espoir, mais surtout la conviction que 1905 mènera à l'instauration de cette structure libérale et constitutionnelle que certains décembristes appelaient de leurs vœux. Le processus révolutionnaire de 1905 n'est pas encore perçu comme un échec - ce sera le cas après la fin de la «période constitutionnelle », lorsque les doutes sur la possibilité d'une transformation de la société russe s'installeront chez de nombreux intellectuels libéraux et radicaux.

\section{Conclusion}

Dès la chute du tsarisme, les recherches et les travaux sur les mouvements d'opposition au tsarisme prennent une importance majeure. Des instituts d'enseignement et d'étude des mouvements révolutionnaires sont créés. Des revues naissent, se spécialisent dans cette thématique, qui s'ancre dans une révision, désormais officielle, de l'histoire russe, perçue sous l'angle des oppositions pluriséculaires au tsarisme.

Or, si 1917 marque un changement important dans la place accordée aux études sur les oppositions au tsarisme, qui ne sont plus en marge des recherches historiques, les années qui précèdent 1917 constituent la genèse de l'historiographie du mouvement révolutionnaire russe. C'est à ce moment que naissent les premières interprétations du mouvement révolutionnaire et les polémiques sur sa filiation. La période soviétique des années 1920 sera profondément marquée par cette genèse historiographique, notamment parce que ce sont en partie les mêmes historiens qui continuent à travailler sur cette thématique après 1917 . Toutefois, dès le « grand tournant » de la fin des années 1920 en Russie, qui affecte tous les domaines de la sphère politique, sociale, économique, artistique et intellectuelle, des changements importants ont lieu dans le domaine des recherches historiques. Les travaux « pionniers » des historiens ayant étudié l'histoire du mouvement de libération russe entre 1905 et 1917 ne sont plus mentionnés, ou alors de façon négative. D’une façon plus générale, les études et surtout la méthodologie des historiens du début du XXe siècle sont de plus en plus vivement critiquées. Jusqu'à la fin de l'époque soviétique, l'interprétation de l'historiographie officielle est la suivante : du milieu des années 1890 environ jusqu'au début du $\mathrm{XX}^{\mathrm{e}}$ siècle, la « science historique russe bourgeoise » - aurait traversé une profonde « crise ${ }^{71}$. Cette crise aurait connu son apogée en 1905-1907

70. Il ne s'agit d'ailleurs pas seulement des historiens. Les décembristes sont souvent cités parmi l'intelligentsia libérale. Ainsi, par exemple, lors de la «campagne des banquets » organisée par l'Union pour la libération, le soir du 14 décembre 1904, jour anniversaire de l'insurrection décembriste, des orateurs font acclamer les «grands patriotes et martyrs » de 1825 . François-Xavier Coquin, La Révolution russe manquée, p. 38.

71. Il existe un grand nombre de publications soviétiques qui analysent la « crise » de l'historiographie russe avant la révolution d'Octobre. On peut mentionner N. L. Rubinštejn, Russkaja istoriografija [L'historiographie russe], M.: Gospolitizdat, 1941 ; A. L. Šapiro, Russkaja 
précisément, la Révolution ayant montré l'impasse dans laquelle se trouvaient les historiens « bourgeois » ou « libéraux ». Après cette date commence « l'étape finale de la crise de l'historiographie russe bourgeoise, qui devait mener à son effondrement suite à la révolution d'Octobre » et à la victoire du communisme ${ }^{72}$. Et pour appuyer leurs dires, les historiens soviétiques citent souvent les propos critiques de N. Pavlov-Sil'vanskij portés sur l'historiographie russe de son époque. Selon eux, Pavlov-Sil'vanskij fut l'un des représentants les plus progressistes de l'historiographie prérévolutionnaire russe. Toutefois, il ne réussit pas à faire «avancer» la science historique de son temps, car il ne rompit jamais clairement avec la « méthodologie bourgeoise de la connaissance historique ${ }^{73}$ et n'adopta pas le « matérialisme historique », la conception marxiste du développement historique, l'unique conception capable de fournir une explication valable à la voie du développement historique russe ${ }^{74}$.

Pourtant, l'historiographie de la fin du XIX siècle et du début du XX $\mathrm{X}^{\mathrm{e}}$ siècle est peut-être la plus féconde de toute l'historiographie russe. Les historiens ont pour la plupart appris leur métier dans les deux grandes «écoles » historiques russes, l'université de Moscou (liée aux noms de Sergej Solov'ev et de Vasilij Ključevskij), et l'université de Saint-Pétersbourg (liée à ceux de Konstantin BestuževRjumin et de Sergej Platonov) ${ }^{75}$, où ils ont bénéficié d'une excellente formation intellectuelle. Dans la Russie du début du Xxe siècle, le niveau des recherches historiques et des réflexions méthodologiques n'a rien à envier à celui de l'Occident. Certains représentants de l'historiographie russe, tels Kareev et Miljukov, s'y sont d'ailleurs faits un nom. La révolution de 1905, en élargissant les recherches historiques à de nouvelles thématiques, en ouvrant l'accès aux documents sur l'histoire sociale et politique du XIX ${ }^{\mathrm{e}}$ siècle, a eu sur elle un impact positif. Elle a en outre amené certains historiens à reformuler et à affiner, lors de polémiques exacerbées par les événements politiques, leurs positions méthodologiques, mais également parfois leur vision de l'histoire russe. Elle a dès lors contribué à enrichir une histo-

istoriografija $v$ period imperializma [L'historiographie russe durant la période de l'impérialisme], L.: Izd. LGU, 1962 ; I. D. Koval’čenko, A. E. Šiklo, « Krizis russkoj buržuaznoj istoričeskoj nauki v konce XIX-načale XX veka » [La crise de la science historique bourgeoise russe à la fin du XIX ${ }^{\mathrm{e}}$ siècle et au début du XXe siècle], Voprosy istorii, $\mathrm{n}^{\circ} 1$, janvier, 1982, etc.

72. Koval’čenko, Šiklo, « Krizis russkoj buržuaznoj istoričeskoj nauki... », p. 33.

73. S. O. Šmidt, I. D. Koval`čenko, S. S. Dmitriev, A. M. Saharov, « O predmete i soderžanii universitetskogo kursa istoriografii istorii SSSR » [À propos du contenu du cours universitaire consacré à l'historiographie de l'histoire de l'URSS], Voprosy istorii, n ${ }^{\circ}$, août, 1963, p. 79.

74. S. O. Šmidt, I. D. Koval`čenko, S. S. Dmitriev, A. M. Saharov, « O predmete i soderžanii... », p. 78.

75. Au sujet de ces deux «écoles » et de l'historiographie russe de la fin du XIX ${ }^{\mathrm{e}}$ siècle et du début du $\mathrm{XX}^{\mathrm{e}}$ siècle, mentionnons entre autres Pavel Miljukov, Vospominanija [Mémoires], M.: Vagrius, 2001, p. 125-135, A. N. Šahanov, Russkaja istoričeskaja nauka vtoroj poloviny XIX - načala XX veka. Moskovskij i Peterburgskij universitety [La Science historique russe de la deuxième moitié du XIX ${ }^{\mathrm{e}}$ siècle et du début du XX $\mathrm{X}^{\mathrm{e}}$ siècle. Les universités de Saint-Pétersbourg et de Moscou], M.: Nauka, 2003 et enfin, E. A. Rostovcev, A. S. Lappo-Danilevskij i peterburgskaja istoričeskaja škola [A. S. Lappo-Danilevskij et l'école historique pétersbourgeoise], Rjazan' : NRII, 2004. 
riographie russe dont l'évolution était remarquable depuis la deuxième moitié du XIX ${ }^{e}$ siècle. Ce n'est pas la révolution de 1905 qui a plongé l'historiographie russe dans une crise profonde, mais le triomphe du stalinisme au début des années 1930, qui a rendu impossibles toute confrontation, tout questionnement, toute polémique historienne, si nécessaires au renouvellement et au développement de la science historique. Tant d'éléments qui, justement, étaient omniprésents durant les années qui ont précédé la révolution de 1917.

Université de Genève, Faculté des lettres

korine.amacher@lettres.unige.ch 